\title{
Model-Based Design and Experimental Validation of Control Modules for Neuromodulation Devices
}

\author{
Hector M. Romero Ugalde, David Ojeda, Virginie Le Rolle, David Andreu, David Guiraud, Jean-L. Bonnet, \\ Christine Henry, Nicole Karam, Albert Hagège, Philippe Mabo, Guy Carrault, and Alfredo I. Hernández*
}

\begin{abstract}
Goal: The goal of this paper is to propose a modelbased control design framework, adapted to the development of control modules for medical devices. A particular example is presented in which instantaneous heart rate is regulated in real-time, by modulating, in an adaptive manner, the current delivered to the vagus nerve by a neuromodulator. Methods: The proposed framework couples a control module, based on a classical PI controller, a mathematical model of the medical device, and a physiological model representing the cardiovascular responses to vagus nerve stimulation (VNS). In order to analyze and evaluate the behavior of the device, different control parameters are tested on a "virtual population," generated with the model, according to the Latin Hypercube sampling method. In particular, sensitivity analyses are applied for the identification of a domain of interest in the space of the control parameters. The obtained control parameter domain has been validated in an experimental evaluation on six sheep. $R e$ sults: A range of control parameters leading to accurate results was successfully estimated by the proposed model-based design method. Experimental evaluation of the control parameters inside such a domain led to the best compromise between accuracy and time response of the VNS control. Conclusion: The feasibility and usefulness of the proposed model-based design method were shown, leading to a functional, real-time closed-loop control of the VNS for the regulation of heart rate.
\end{abstract}

Index Terms-Heart rate, model-based control design, modeling, simulation, vagus nerve stimulation.

\section{INTRODUCTION}

V AGUS nerve stimulation (VNS) offers an alternative therapy in various pathologies, such as epilepsy, supraventricular arrhythmias, and heart failure [1]. VNS therapy is commonly delivered in an open-loop approach, with fixed

This work was supported by BPIFRANCE within the framework of the French "Investments for the future" program-INTENSE project. Asterisk indicates corresponding author.

${ }^{*}$ A. I. Hernández is with INSERM, U1099, Rennes, F-35000, France, and also with Université de Rennes 1, LTSI, Rennes, F-35000, France (e-mail: alfredo.hernandez@inserm.fr).

H. Romero, D. Ojeda, V. Le Rolle, and G. Carrault are with INSERM, U1099, Rennes, F-35000, France, and also with Université de Rennes 1, LTSI, Rennes, F-35000, France.

D. Andreu and D. Guiraud are with the INRIA Sophia Antipolis, and with the DEMAR Project and LIRMM, University of Montpellier II.

J. L. Bonnet and C. Henry are with Sorin Group, Clamart, France.

N. Karam and A. Hagège are with the INSERM, UMR970 Paris Cardiovascular Research Center, with the Assistance Publique-Hôpitaux de Paris, Department of Cardiology, Hôpital Européen Georges Pompidou, and also with Paris Descartes University, PRES Paris Sorbonne.

P. Mabo is with the CHU Rennes, Department of Cardiology, with INSERM, CIC 1414, Rennes, F-35000, France, with INSERM, U1099, Rennes, F-35000, France, and also with Université de Rennes 1, LTSI, Rennes, F-35000, France. stimulation parameters, without any feedback regulation. However, it is now widely accepted that closed-loop control may be necessary to optimize the response to the therapy in an adaptive manner and minimize side effects of neurostimulation devices [2], [3].

A variety of physiological data may be employed as control variables in a closed-loop approach, depending on the target function of VNS control. Previous published studies have proposed methods to control heart rate, since this variable is particularly important and is readily observable [3]-[6].

In [7], a control system, based on the classical cumulative sum control chart technique, adjusts the stimulation frequency in order to regulate the average ventricular rate during atrial fibrillation. In the systems proposed by Zhang et al. [8] and by Tosato et al. [3], a proportional-integral (PI) controller was used to modulate respectively the instantaneous amplitude of the impulses applied for VNS and the stimulation frequency. However, due to the difficulty of observing physiological variables in vivo and the complexity and nonlinearity of the underlying physiological mechanisms, traditional control design methods, usually based on the analysis of harmonic responses, cannot be applied in this context. Although the appropriate definition of the controller parameters is of primary importance, especially in the case of a medical device, the control design phase is usually simple and based on heuristics. Novel methods for control design should, thus, be proposed.

Model-based design (MBD) has already been used as a way to overcome the difficulties associated with complex control systems [9] and has been widely used for designing and testing realtime control applications in a variety of contexts, such as the automotive field [10], space research [11], renewable energies [12] or the aerospace field [13]. This method is generally based on four phases: $i$ ) create a model of the plant (system of interest and actuators), ii) define the controller's mathematical structure, iii) couple the model and the controller, $i v$ ) apply an analysis phase, to identify the optimal parameters of the controller by performing recurrent simulations of the coupled system. Nevertheless, the application of such an MBD scheme is particularly difficult in the biomedical context, due to the complexity and low observability associated with the underlying physiological systems.

In this paper, we propose a novel MBD framework particularly adapted for the design of control modules in medical devices. Although the framework is completely general, the example of the design of an optimal closed-loop control system for the VNS will be developed. Section II describes the proposed framework, including the underlying models and the retained control structure. An original sensitivity analysis approach, based on the creation of "virtual populations" and a 


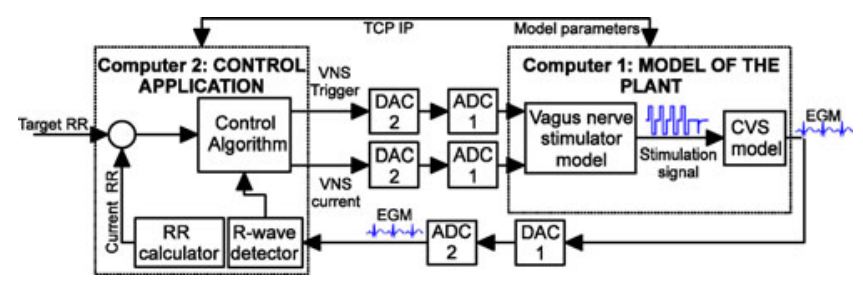

Fig. 1. Model-based control design framework. The control application and the model of the plant are coupled through analog signals.

fully crossed design, is proposed in Section III for the optimization of the controller parameters. An experimental validation method is proposed in Section IV in order to evaluate the control parameters obtained from the analysis phase. Section V presents the results of the analysis and animal experimental validation on six sheep. Sections VI and VII propose, respectively, the discussion and conclusions of this study.

\section{PROPOSED MBD FrAMEWORK}

The proposed MBD framework (see Fig. 1) is composed of: i) the model of the plant, implemented in computer 1, which integrates a model of the neuromodulator and a model of the cardiovascular response to the VNS, ii) the control application, implemented in computer 2, which includes a signal-processing module and a control algorithm (PI), iii) two data acquisition (DAQ) devices NI USB-6211 and iv) a TCP/IP communication link, which allows computer 2 to change the model parameters on computer 1 .

The model synthesizes intracardiac ElectroGraM (EGM) signals which are sent to the control application through a digital to analog converter in computer 1 (DAC1) and an analog to digital converter in computer 2 (ADC2). EGMs are processed for real-time detection of R-waves and to compute the current RR interval, which is compared to a target RR, defined by the user. An error computed between the instantaneous and target RR intervals is presented as input to the proposed control algorithm, which determines the appropriate VNS current amplitude. As a difference from previous studies [3], [7], VNS in this study is performed synchronously with cardiac beats. Therefore, the controller algorithm also determines the instant of VNS activation, using the currently detected $\mathrm{R}$-wave instant as a reference. This VNS activation instant is transmitted to the model via an analog signal (VNS Trigger), presenting a squared pulse that triggers VNS on the neuromodulator model. The computed current amplitude and trigger signals are sent to the neurostimulator model in computer 1 via different channels of DAC2 and ADC1.

The main interest of coupling these two computers through analog signals is that, once the parameters of the controller have been optimized, we can connect computer 2 directly to the real neurostimulator and a real sheep to perform in vivo validation.

\section{A. Model of the Plant}

In order to represent the plant, three different sub-models have been created and coupled (see Fig. 2): 1) the cardiovascular

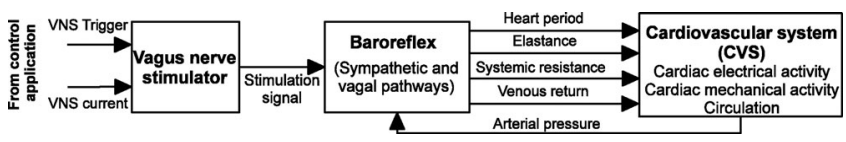

Fig. 2. Representation of the global structure of the model, including the VN stimulator, the baroreflex, and the CVS.

system (CVS), 2) the baroreflex, including vagal activity, and 3) the vagus nerve $(\mathrm{VN})$ stimulator (actuator).

1) Model of the CVS: This model is based on previous studies of our group [14]-[18] and has been adapted using experimental data acquired from different sheep. It is constituted of two coupled components including lumped-parameter representations of: 1) the cardiac electrical activity, 2) the cardiac mechanical activity, and 3) the circulation.

The proposed model of the cardiac electrical activity, is based on a set of coupled automata [15], [16]. Each automaton represents the electrical activation state of a given myocardial tissue, covering the main electrophysiological activation periods: slow diastolic depolarization (SDD), upstroke depolarization (UD), absolute refractory, and relative refractory periods. The whole simplified model consists of six automata representing: the sinoatrial node, both atria, the atrioventricular node, upper bundle of His, lower bundle of His, and both ventricles. The slope of the SDD of the sinoatrial node is determined by the chronotropic response of the baroreflex model, explained later. The electrical activation of the automata is used to synthesize an EGM, from which a set of parameters can be extracted, such as the instantaneous heart rate.

The cardiac mechanical activity is represented by means of a classical elastance-based approach. For each cardiac chamber, volumes $(V)$ are computed from the integral of their respective net flow. Blood pressure $(P)$ is then calculated from its volume using two pressure-volume relationships associated with systole and diastole, respectively. A periodic function $e(t)$ drives the transition between the systolic $\left(P_{\mathrm{es}}\right)$ and diastolic $\left(P_{\mathrm{ed}}\right)$ relationships as follows:

$$
\begin{aligned}
P(V, t) & =e(t) P_{\mathrm{es}}(V)+(1-e(t)) P_{\mathrm{ed}}(V), \\
P_{\mathrm{es}}(V) & =E_{\mathrm{es}} \cdot\left(V-V_{d}\right), \\
P_{\mathrm{ed}}(V) & =P_{0} \cdot\left(e^{\lambda\left(V-V_{0}\right)}-1\right), \\
\quad e(t) & =A \cdot \exp \left(-B \cdot\left(\left(t-t_{s}\right)-C\right)^{2}\right) .
\end{aligned}
$$

In these equations, the systolic elastance $\left(E_{\text {es }}\right)$ and dead volume $\left(V_{d}\right)$ represent the slope and intercept of the linear relationship of pressure and volume during systole. During diastole, this relationship is nonlinear [19] and described by the diastolic elastance $\left(P_{0}\right)$ and an exponential function of $V$ and the volume at zero pressure $\left(V_{0}\right)$. The diastolic and systolic dynamics are driven by a Gaussian function (4) described by its amplitude $(A)$, width $(B)$, and center $(C)$. The onset of the cardiac cycle, denoted $t_{s}$, is determined by the activation instant (start of the UD period) of the corresponding chamber in the cardiac electrical model presented previously.

Finally, we used the minimal cardiovascular model proposed by Smith et al. [19] for the representation of blood flow dynamics of the CVS. This lumped-parameter model represents the 


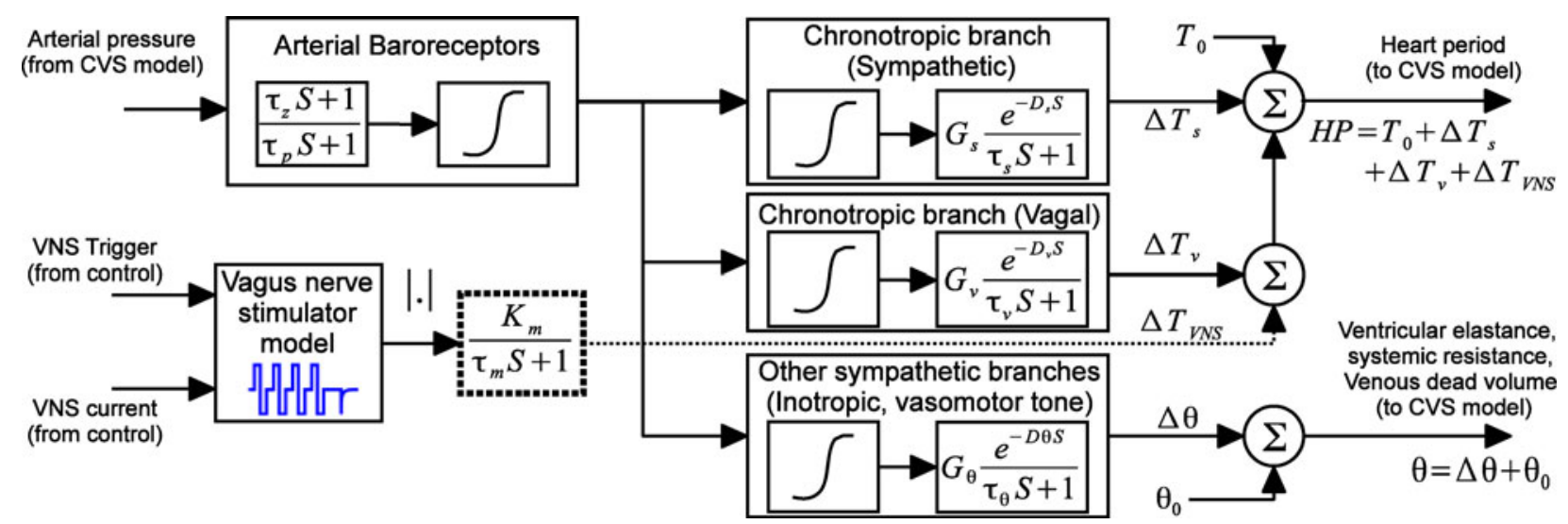

Fig. 3. Representation of the baroreflex model and the model of the VN stimulator. The coupling between these two models is represented by the transfer function in dotted lines.

main features of system-level fluid dynamics using an hydroelectric analogy and has been already studied in our previous studies [17], [18], [20], [21]. Pressure on the circulation chambers are calculated as a linear relationship of their volume and its vascular elastance, following (2). These pressures are then used to calculate flows between chambers as $Q=\frac{\Delta P}{R}$, where $\Delta P$ is the pressure gradient of two chambers and $R$ is the corresponding vascular resistance connecting them (either $R_{\mathrm{sys}}$ or $\left.R_{\text {pul }}\right)$.

2) Baroreflex Model: The baroreflex model, presented in more detail in Fig. 3, was adapted from [22]. Its input variable is the arterial pressure generated by the CVS model. This input is presented to the arterial baroreceptors, represented as a transfer function chained with a saturation function [22]. The efferent pathways are represented as a set of first-order transfer functions describing the linear responses giving as output the modifications of the RR interval (chonotropic effect) through the sympathetic and vagal branches, and modifications of other cardiac functions (inotropism, vasomotor tone, etc.), mainly through sympathetic pathways. This study will be focused on the chronotropic effect, for which the vagal and sympathetic responses are combined linearly as $\mathrm{RR}=T_{0}+\Delta T_{v}+\Delta T_{s}+\Delta T_{\mathrm{VNS}}$, where $T_{0}$ is the intrinsic heart period and $\Delta T_{\mathrm{VNS}}$ is the VNS effect on HR.

3) Model of the Vagus Nerve Stimulator: A model of a VNS device has been developed. This model generates a stimulation pulse train, triggered by an R-wave, and characterized by a given VNS configuration which is defined by a set of adjustable parameters. Pulses are delivered at a programmable preset delay $\left(\mathrm{VNS}_{\text {delay }}\right)$, measured from the instant of R-wave detection for beat $b\left(R_{d e t}^{b}\right)$. Only some cardiac cycles may be stimulated, according to a defined ratio $\left(\mathrm{VNS}_{\text {ratio }}\right)$. The other parameters are: VNS current $(\mathrm{mA})$, pulse width $(\mathrm{ms})$, pulse train frequency ( $\mathrm{VNS}_{\text {freq }}$ in $\mathrm{Hz}$ ), and number of pulses ( $N_{\text {pulses }}$ in Num).

The stimulation signal is coupled to the model of the VN through the transfer function depicted in Fig. 3 with dotted lines. This kind of transfer function has already been used to represent the modulation of heart rate as a function of neural traffic on the VN [23]. As it can be seen on Fig. 3, the output of this transfer function ( $\Delta T_{\mathrm{VNS}}$ ) is added to the contributions of $\Delta T_{v}$ and $\Delta T_{s}$ to calculate RR.

\section{B. Control Design}

As previously mentioned, one of the difficulties of the proposed control approach is that it should work synchronously with the heart period. The controller will be thus updated for each beat $b$, during the time interval $\left[R_{\text {det }}^{b}, \ldots, R_{\text {det }}^{b}+\right.$ $\left.\mathrm{VNS}_{\text {delay }}\right]$. In our current prototype, the two electrodes (the cuff used for VNS and the intracardiac lead) share a common ground terminal. A cross-talk phenomenon between both channels can, thus, arise while stimulating. In order to avoid this crosstalk, the cardiac amplifier is disabled (blanking mode) during VNS. This blanking period is minimized by defining the $\mathrm{VNS}_{\text {delay }} \in$ $[0,100] \mathrm{ms}$, corresponding to the cardiac depolarization phase.

The retained control method is based on a classical PI controller, since previous studies have shown that a PI controller yields better results than a PID controller in the context of VNS [3]. The VNS current to be delivered during beat $b(u(b))$ is thus given by

$$
\begin{aligned}
& u(b)=k_{p}\left(\epsilon(b)+\frac{1}{T_{i}} \sum \epsilon(b)\right) \\
& \epsilon(b)=\mathrm{RR}_{T}-\mathrm{RR}_{O}(b)
\end{aligned}
$$

where $T_{i}$ and $k_{p}$ represent, respectively, the integral and the proportional parameters of the PI controller; $\mathrm{RR}_{T}$ is the target $\mathrm{RR}$, set by the user, and $\mathrm{RR}_{O}(b)$ is the observed $\mathrm{RR}$ interval, computed by the RR calculator module in computer 2 between beats $b-1$ and $b$.

\section{SEnSitivity ANALYSIS}

\section{A. Creation of a "Virtual Population" Based on the Model}

In a previous study [18], we performed a sensitivity analysis on the CVS model described in Section II-A, in order to pinpoint the parameters producing significant effects on heart rate. Here, five highly-sensitive parameters are modified to represent a set of $L=30$ "virtual sheep": the gain $T_{v}$ and time constant $\tau_{v}$ of the chronotropic vagal response, the systemic resistance $R_{\mathrm{sys}}$, the gain of the chronotropic sympathetic response $T_{s}$, and the gain of the VNS effect $K_{m}$. Thirty different parameter vectors (one for each virtual individual) have been generated according to the Latin Hypercube sampling (LHS) method [24], [25]. 


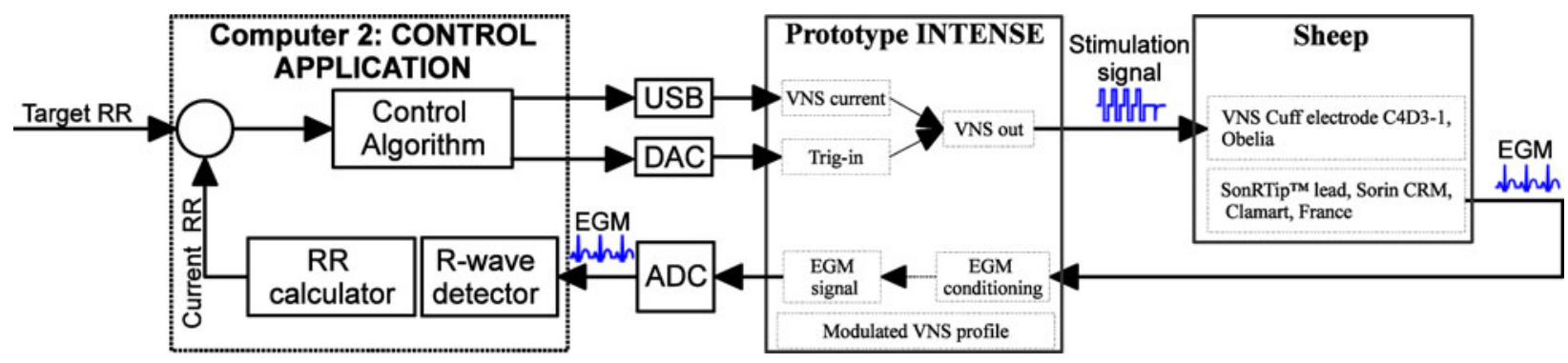

Fig. 4. Implemented VNS closed-loop control system. The control application is the same used in Fig. 1. The model of the stimulator and the plant were simply substituted by the real stimulator and a real sheep.

LHS allows us to spread the parameter values evenly across a predefined range, defined here at $\pm 10 \%$ of the baseline model parameter values, partitioning each input distribution into intervals of equal probability, and selecting one sample from each interval. Moreover, LHS ensures no correlation between the inputs. Once the virtual population was generated, the PI controller is applied to each individual, using different controller parameters, as described in the next section.

\section{B. Sensitivity Analysis of the Model-Control Framework}

The objective of the sensitivity analysis on the controller parameters is to find a domain on the parameter space $\left(T_{i}, k_{p}\right)$, providing the best performance on the whole virtual population. The proposed MBD framework is thus applied to perform a fully crossed design (full factorial experiment), consisting in testing all the combinations of $T_{i}$ and $k_{p}$, within a given range, and then analyzing the performance obtained by the application of these values to the whole virtual population. In this experiment, 25 values of $T_{i}$ and $k_{p}$, into the ranges $T_{i} \in[0.05,3]$ and $k_{p} \in$ $[0.0001,0.12]$, were used. Based on previous results [26], these ranges of $T_{i}$ and $k_{p}$ were chosen to cover a large exploration of the parameter space, while preserving acceptable performance.

For each combination of $\left(T_{i}, k_{p}\right)$, the controller (computer 2) sends, via TCP/IP, a set of model parameters, corresponding to one virtual sheep. The combination is tested for each sheep of the virtual population. The control test consists in setting the controller off for 100s and then turning it on for 100s. The following performance indicators, were computed each time the controller is turned $\mathrm{ON}$ :

1) The mean squared error (MSE) calculated from the beat in which the system arrived to the steady state $\left(b_{s}\right)$ to $b_{s}+N$ :

$$
\operatorname{MSE}=\frac{1}{N} \sum_{b=b_{s}}^{b_{s}+N} \epsilon^{2}(b) .
$$

2) The percent overshoot (\%OS) that corresponds to the amplitude of the overshoot response, expressed as a percentage of the steady-state value.

3) The rise time $T_{r}$ required for the waveform to go from $10 \%$ of the final value to $90 \%$ of the final value.

Finally, the following linear combination of the three performance indicators was proposed in order to provide a global criterion, to be used in the selection of a relevant range of control parameters $\left(T_{i}, k_{p}\right)$

$$
\mathrm{F}=0.25 \frac{\overline{\mathrm{MSE}}}{\max (\overline{\mathrm{MSE}})}+0.25 \frac{\overline{\% \mathrm{OS}}}{\max (\overline{\% \mathrm{OS}})}+0.5 \frac{\overline{T_{r}}}{\max \left(\overline{T_{r}}\right)}
$$

where $\overline{\mathrm{MSE}}, \overline{\% \mathrm{OS}}$, and $\overline{T_{r}}$ are the mean values of the performance indicators on the virtual population. Notice that by using this selection criteria, we give the same importance to the accuracy (MSE, \%OS) and speed of convergence $T_{r}$.

\section{EXPERIMENTAL VALIDATION OF THE CONTROLLER}

The ranges of $T_{i}$ and $k_{p}$ defined from the above sensitivity analysis were experimentally validated on six sheep under two different anesthetic agents.

\section{A. Experimental Protocol}

Six sheep were included in this experimental study, which was conducted under the approval issued by the French ethics committee for animal experimentation. A bipolar pacemaker lead containing an intracardiac accelerometer (SonRTip lead, Sorin CRM, Clamart, France) was placed in the right ventricle and a cuff-type VNS electrode (Cuff electrode C4D3-1, Obelia) was implanted on the right $\mathrm{VN}$, at a cervical site. The aortic artery dissection is realized at an equal distance between the head and the trunk. The VN is gently removed from the aortic sheath and the lead is placed around the VN. These leads were connected to the proposed PI control system, as discussed in the next section.

During the intervention, sheep were initially anesthetized with Propofol and a morphine bolus. After this initial phase, the sheep were anesthetized by Etomidate $(100 \mu \mathrm{g} / \mathrm{kg} / \mathrm{min})$. Then, after a verification stage of the implanted instrumentation (in particular VNS electrode impedance and EGM quality), the experimental evaluation of the proposed control system was performed. Finally, the sheep were anesthetized by Isoflurane, in order to test the same controller on a same sheep, but under different autonomic conditions. The surface ECG, the EGM, the left intraventricular pressure, and the body temperature were monitored during the whole procedure. Breathing was artificially controlled at $0.3 \mathrm{~Hz}$ (18 breaths/min).

\section{B. PI Closed-Loop Control System Implementation}

The closed-loop control system, depicted in Fig. 4, is composed of: $i$ ) the bipolar EGM sensor, ii) the cuff electrode used 


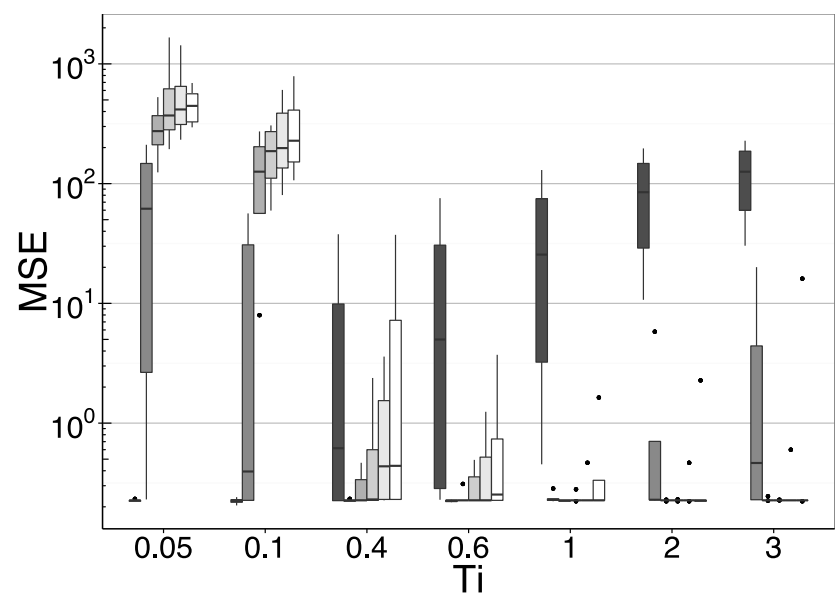

(a)

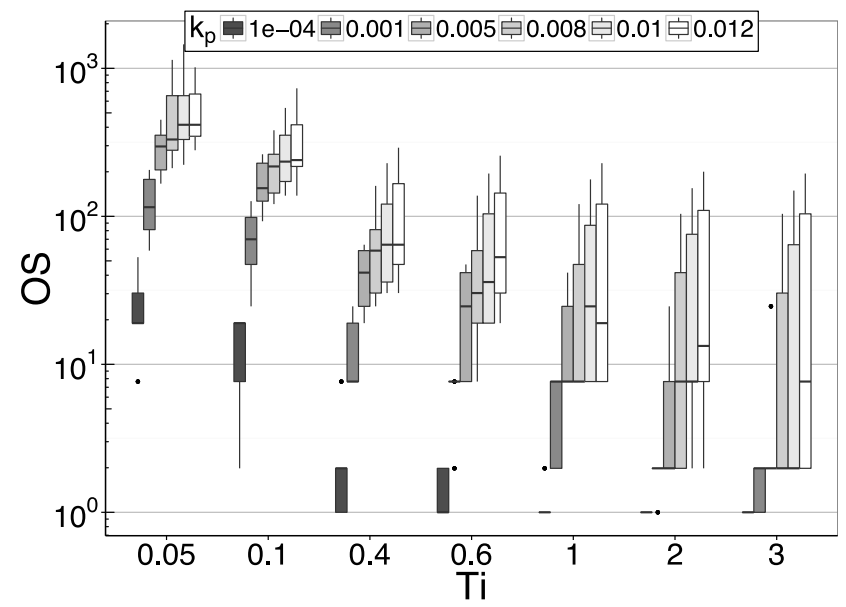

(c)

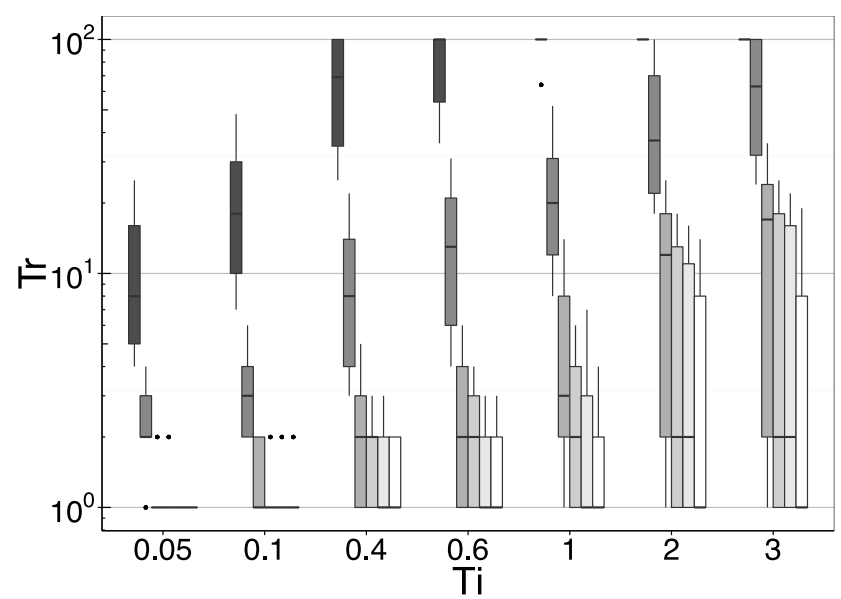

(b)

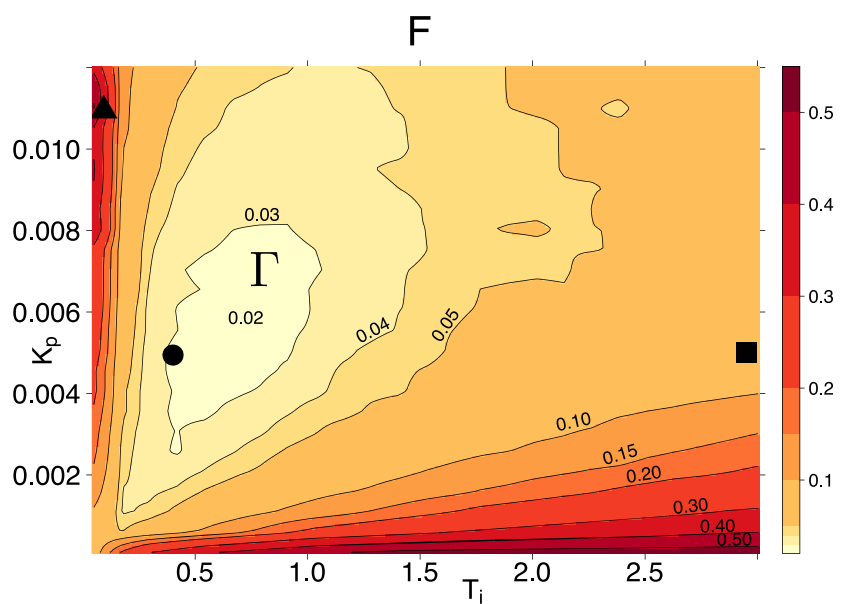

(d)

Fig. 5. Boxplots presenting the sensitivity analysis results for $25 \times 25$ combinations of $\left(T_{i}, k_{p}\right)$ on the whole virtual population in terms of MSE (A), $T_{r}$ (B), and OS (C). Representation of the values of function F on the space of parameters $\left(T_{i}, k_{p}\right)$ (D). The symbols $\boldsymbol{\Lambda}, \bullet$ and $\mathbf{\square}$ represent three different ( $\left.T_{i}, k_{p}\right)$ configurations, shown in Fig. 6.

for VNS, iii) a custom prototype external neurostimulator (Prototype INTENSE v1.2), able to drive up to 24 channels of stimulation in accurate synchronization, with a programmable distribution of currents over the channels, allowing for complex temporal stimulation waveforms [27], iv) a DAQ device NI USB-6211, and 5) a real-time control application, which receives and process the EGM signal in real time and implements the VNS control loop system.

The control application is the same used for the sensitivity analysis phase, during which it was coupled with the model. The interest of the proposed approach is that it allows us to pass from the simulation-based evaluation to the real experimental evaluation, with minimal effort and keeping the same control components. Indeed, for the experimental evaluation, the model of the stimulator and the plant of the proposed MBD framework, shown in Fig. 1, were simply substituted by the real stimulator (Prototype INTENSE v1.2) and the real plant (sheep). The only difference is that the control protocol to modify the VNS amplitude is sent to the real stimulator via USB communication, and the instruction to stimulate with the new VNS parameter is sent to the Trig-in input of the stimulator via the analog output of the DAQ NI 6211.

\section{RESULTS}

\section{A. Sensitivity Analysis of $T_{i}$ and $k_{p}$ Evaluated on the Model}

Fig. 5 (a), (b) and (c) shows boxplots obtained for all combinations $\left(T_{i}, k_{p}\right)$ on the virtual population. For most parameter values of $k_{p}$, an increase of $T_{i}$ is associated with an improvement of the accuracy (MSE and \%OS ), while a rise of $k_{p}$ induces an decrease of precision and rapidity. However, the system response to $T_{i}$ and $k_{p}$ is more complex to analyze, since it depends on the combination of both parameters.

The combined function F is exposed in Fig. 5(D), as a function of $T_{i}$ and $k_{p}$. Low values of $T_{i}$ and $k_{p}$ are associated with poor performances. An optimized set of parameters $\Gamma$ appears for the restrictive condition corresponding to $\mathrm{F}<0.02$ :

$$
\Gamma=\left\{\left(T_{i}, k_{p}\right), T_{i} \in[0.4,0.6], k_{p} \in[0.004,0.006]\right\} .
$$

Fig. 6 shows the dynamic response of RR intervals (A) and the applied current $(\mathrm{B})$, when the $\left(T_{i}, k_{p}\right)$ parameters are set to values inside and outside $\Gamma$. Low values of $T_{i}$ are associated with unacceptable oscillations, while elevated $T_{i}$, combined with a moderate value of $k_{p}$, yields much longer time response. On 


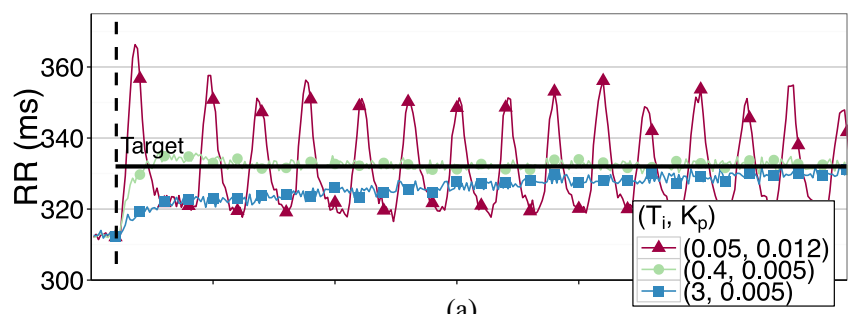

(a)

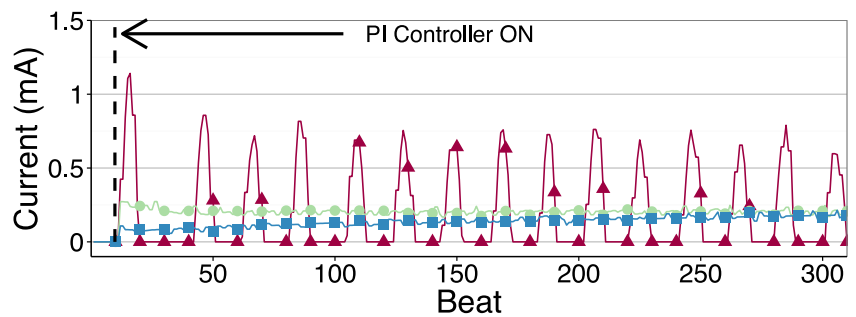

(b)

Fig. 6. Evaluation of three different combinations of $\left(T_{i}, k_{p}\right)$, corresponding to symbols $\boldsymbol{\Lambda}, \bullet$ and $\boldsymbol{\square}$ on Fig 5, applied on a single virtual sheep, showing the instantaneous RR response (A) and the VNS current obtained as output of the PI controller (B).

the contrary, the values inside $\Gamma$ yield a satisfying compromise between accuracy and time response.

\section{B. Experimental Validation}

The controller was tested on six sheep, three of them anesthetized by two different anesthetics (Etomidate and Isoflurane). In order to validate the ranges of $T_{i}$ and $k_{p}$ derived from the previous MBD, and since the animal experimentation time is limited, two approaches with a reduced set of combinations of $T_{i}$ and $k_{p}$ were studied. For the first sheep, $k_{p}$ was fixed to $k_{p}=0.005 \in \Gamma$ and several values of $T_{i}$ are explored. The target value was set at $110 \%$ of the baseline RR, measured at the beginning of the experimentation, so as to observe the convergence response for large $T_{i}$ values $\left(T_{i}>1\right)$. For all other sheep, different values of $k_{p}$ are used, while fixing $T_{i}=0.4 \in$ $\Gamma$. RR target values were set at $105 \%$ and $110 \%$ of the baseline $\mathrm{RR}$, but only results leading to attainable target values (without premature ventricular contractions) were kept. Due to technical reasons (recording problems), performance measures for sheep 5 at $105 \%$ were not computed.

1) Experimental Validation of $T_{i}$ : The sheep was anesthetized by Isoflurane. The target RR is set at $110 \%$ of the RR baseline and five values of $T_{i}$ were tested. Fig. 7 shows that an increase of $T_{i}$ is associated with reduced oscillations but also with a reduced speed of convergence. These results are in concordance with the sensitivity analysis presented in Fig. 5. Note that the combination $\left(T_{i}=0.4, k_{p}=0.005\right) \in \Gamma$ yields the best compromise between precision and speed of convergence. Results given in Table I (see Sheep 1) confirm these conclusions.

2) Experimental Validation of $k_{p}$ : Sheep 2 was anesthetized by Etomidate. Results are presented for a target RR set at $105 \%$ of the RR baseline, with $T_{i}$ fixed at 0.4 , and five values of $k_{p}$. Fig. 8 shows that, in all cases, the target is reached. As expected, when $k_{p}$ is increased, the speed of convergence is increased, but MSE and \%OS are increased too (see Table I, Sheep 2). It

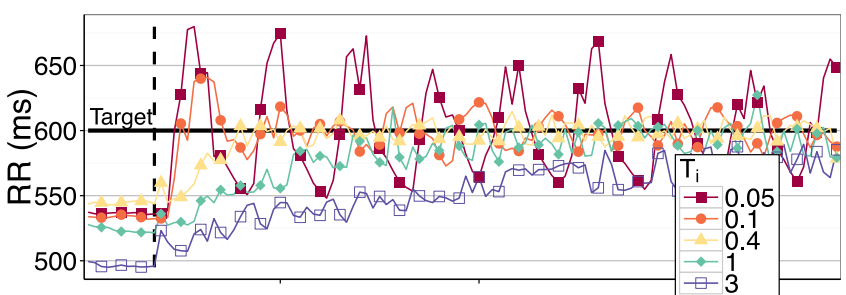

(a)

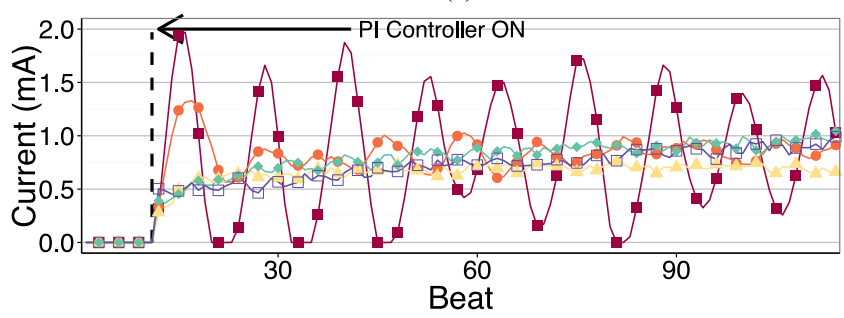

(b)

Fig. 7. Experimental results on sheep 1 anesthetized by Isoflurane with a target set at $110 \%$ of the RR baseline. Five values of $T_{i}$ were tested, while observing the instantaneous RR interval of the sheep (A) and the VNS current obtained from the controller (B).

TABLE I

Performance Measures on ANimal Experimentation; A = ANESTHETICS, $\mathrm{I}=$ ISOFLURANE, $\mathrm{E}=$ ETOMIDATE

\begin{tabular}{|c|c|c|c|c|c|c|c|c|}
\hline Sheep & A & Target & $k_{p}$ & $T_{i}$ & MSE & $\% \mathrm{OS}$ & $T_{r}$ & $\begin{array}{c}\text { Baseline RR } \\
\text { (mean } \pm \text { std) }\end{array}$ \\
\hline \multirow[t]{5}{*}{1} & \multirow[t]{5}{*}{ I } & \multirow[t]{5}{*}{$110 \%$} & \multirow[t]{5}{*}{0.005} & 0.05 & 909.32 & 125.60 & 4 & $537.90 \pm 1.09$ \\
\hline & & & & 0.1 & 108.31 & 61.32 & 5 & $534.10 \pm 1.50$ \\
\hline & & & & 0.4 & 53.97 & 26.24 & 14 & $544.16 \pm 1.36$ \\
\hline & & & & 1 & 149.73 & 23.83 & 31 & $525.35 \pm 1.52$ \\
\hline & & & & 3 & 1000.45 & 0 & - & $499.17 \pm 2.00$ \\
\hline \multirow[t]{5}{*}{2} & \multirow[t]{5}{*}{ E } & \multirow[t]{5}{*}{$105 \%$} & 0.012 & \multirow[t]{5}{*}{0.4} & 246.10 & 152.87 & 1 & $653.70 \pm 0.76$ \\
\hline & & & 0.008 & & 99.32 & 95.67 & 3 & $659.57 \pm 0.83$ \\
\hline & & & 0.005 & & 51.92 & 81.08 & 7 & $665.01 \pm 0.95$ \\
\hline & & & 0.001 & & 13.35 & 11.53 & 51 & $663.68 \pm 1.01$ \\
\hline & & & 0.0001 & & 1.01 & 0 & 81 & $652.03 \pm 0.53$ \\
\hline \multirow[t]{4}{*}{3} & \multirow[t]{2}{*}{ E } & \multirow[t]{2}{*}{$105 \%$} & 0.005 & \multirow[t]{4}{*}{0.4} & 198.67 & 145.20 & 12 & $426.25 \pm 0.97$ \\
\hline & & & 0.001 & & 42.90 & 50.39 & 63 & $420.96 \pm 1.27$ \\
\hline & \multirow[t]{2}{*}{ I } & \multirow[t]{2}{*}{$105 \%$} & 0.005 & & 392.97 & 144.49 & 1 & $549.68 \pm 0.98$ \\
\hline & & & 0.001 & & 157.53 & 56.35 & 67 & $549.64 \pm 1.01$ \\
\hline \multirow[t]{2}{*}{4} & \multirow[t]{2}{*}{ E } & \multirow[t]{2}{*}{$105 \%$} & 0.01 & \multirow[t]{2}{*}{0.4} & 56.68 & 35.51 & 17 & $672.17 \pm 4.72$ \\
\hline & & & 0.005 & & 88.58 & 23.15 & 39 & $672.71 \pm 5.36$ \\
\hline \multirow[t]{6}{*}{5} & \multirow[t]{4}{*}{ E } & \multirow[t]{2}{*}{$105 \%$} & 0.005 & \multirow[t]{6}{*}{0.4} & 75.46 & 101.39 & 19 & $400.03 \pm 1.14$ \\
\hline & & & 0.001 & & 33.36 & 90.12 & 91 & $399.91 \pm 1.12$ \\
\hline & & \multirow[t]{2}{*}{$110 \%$} & 0.005 & & 780.10 & 133.01 & 8 & $400.10 \pm 1.15$ \\
\hline & & & 0.001 & & 133.60 & 6.02 & 56 & $400.44 \pm 1.02$ \\
\hline & \multirow[t]{2}{*}{ I } & \multirow{2}{*}{$110 \%$} & 0.005 & & 838.60 & 92.61 & 9 & $578.52 \pm 1.23$ \\
\hline & & & 0.001 & & 116.60 & 5.96 & 48 & $581.23 \pm 2.08$ \\
\hline \multirow[t]{8}{*}{6} & \multirow[t]{4}{*}{$\mathrm{E}$} & \multirow[t]{2}{*}{$105 \%$} & 0.005 & \multirow[t]{8}{*}{0.4} & 685.49 & 26.37 & 7 & $595.30 \pm 1.01$ \\
\hline & & & 0.001 & & 5628 & 7.54 & 26 & $598.12 \pm 1.17$ \\
\hline & & $110 \%$ & 0.005 & & 61.18 & 7.91 & 7 & $597.53 \pm 0.94$ \\
\hline & & & 0.001 & & 21.03 & 4.63 & 44 & $598.30 \pm 0.83$ \\
\hline & I & $105 \%$ & 0.005 & & 105.16 & 10.35 & 8 & $693.41 \pm 0.68$ \\
\hline & & & 0.001 & & 124.80 & 8.85 & 40 & $687.57 \pm 0.92$ \\
\hline & & $110 \%$ & 0.005 & & 25.92 & 5.02 & 14 & $694.87 \pm 1.23$ \\
\hline & & & 0.001 & & 23.04 & 4.75 & 54 & $691.40 \pm 1.16$ \\
\hline
\end{tabular}

is interesting to note that, among the tested values, the value $k_{p}=0.005 \in \Gamma$, yields the best compromise between speed of convergence $\left(T_{r}\right)$ and accuracy (MSE and \%OS).

3) Global Experimental Results: Table I, presents the experimental results on all the sheep included in our experimental 


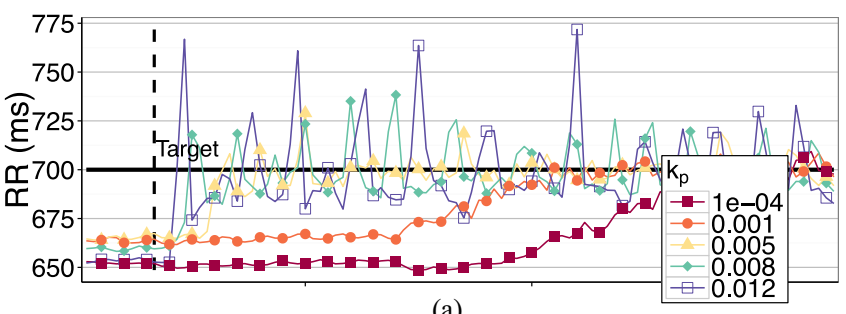

(a)

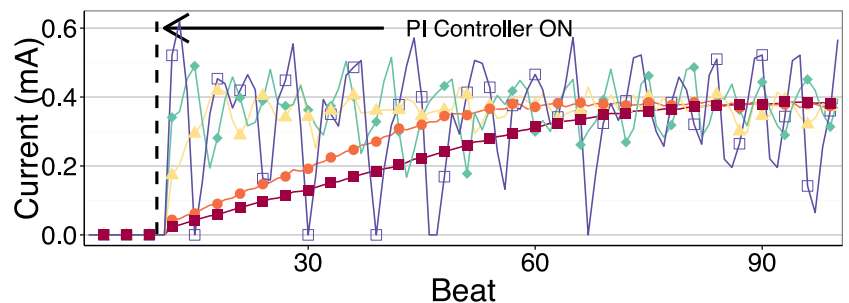

(b)

Fig. 8. Experimental results on sheep 2, anesthetized by Etomidate with a target set at $105 \%$ of the RR baseline. Five values of $k_{p}$ were tested, while observing the instantaneous RR interval of the sheep (A) and the VNS current obtained from the controller (B).

evaluation, using different targets and control parameter values. Note that the use of $k_{p}$ values higher than 0.012 (i.e., the maximum value of $k_{p}$ analyzed on the virtual population) was avoided during the experimentation, after sheep 2, in order to ensure a safety condition. In all cases, the expected performance is achieved using PI parameters that are inside the domain $\Gamma$, derived from the MBD approach. Table I also presents the baseline values of RR interval. No correlation was found between baseline RR values and the obtained performance indicators, showing the controller's ability to adapt to various conditions.

\section{DISCUSSION}

Previous studies have shown the utility of using a theoretical approach in order to improve the design of medical devices. For instance, in [28], a first-order transfer function is used to represent the relationship between the current delivered to a neuron (input of the controlled system) and the interspike intervals of the neuron (output of the controlled system). In [3], the relationship between the VNS frequency (input of the controlled system) and the RR interval (output of the controlled system) is modeled by a first-order transfer function. The PI controllers designed in both papers work successfully in a limited range. However, the authors suggest that better results may be reached by using more detailed models, which can take into account the more complex (and often nonlinear) function of the underlying system. As a difference to these previous studies, the MBD presented in our paper is based on an integrated physiological model including nonlinear dynamics and relies on a complete sensitivity analysis of this model. Therefore, the designed PI controller may perform better in a larger range, since nonlinearities and physiological delays are taken into account. Moreover, a safer behavior is ensured because the controller was tested on a large virtual population, obtained from significant variations of the model parameter values, and leading to a large variety of responses to VNS. To our knowledge, this paper is the first to describe such a model-based approach, based on an integrated model and including the creation of a virtual population associated with an exhaustive sensitivity analysis of control parameters.

Concerning the particular application developed in this paper for closed-loop VNS therapy, the difficulty is to find an optimal set of PI parameters presenting the best compromise between time of convergence and accuracy, while assuring safe stimulation conditions and smooth transitions on the current applied to the nerve. For instance, we can observe in Figs. 7 and 8(B) that the applied current during VNS tends to 1 and 0.4 $\mathrm{mA}$, respectively. However, the dynamics of the VNS current depend directly on the control parameters (Figs. 7 and 8(B)). Low $\left(k_{p}\right)$ and high $\left(T_{i}\right)$ are associated with slow, low-amplitude dynamics of the VNS current, while high $\left(k_{p}\right)$ and low $\left(T_{i}\right)$ provoke rapid, high-amplitude changes on the applied current, and oscillations in the observed RR interval. The set of control parameter values, identified through the sensitivity analysis phase within the $\Gamma$ domain, lead to an appropriate adjustment on VNS current dynamics in the experimental evaluation, provoking suitable changes on the observed RR interval, and a good compromise between accuracy and speed of convergence. Moreover, the proposed PI controller provides satisfactory results for both anesthetic agents, Isoflurane and Etomidate (see I and $\mathrm{E}$ in Table I, respectively), that are associated with two different autonomic states. This finding is particularly interesting for the use of the proposed controller in clinical practice, since the autonomic states of a patient could be affected by several long and short-term influences. Furthermore, since most of the control parameter values used during experimental evaluation were kept within the $\Gamma$ domain, the evaluated PI controllers could ensure a safe and optimized response for a majority of circumstances.

Limitations of this study are mainly related to the experimentation phase. Although the experimental conditions were standardized (anesthesia, regulated breath,...), the uncontrolled variations of environmental conditions (evolution of the anesthetic's effect during the experimentation, physiological variations, ...) made the RR baseline value evolves through time, even for the same sheep under the same anesthetic agent (Table I, last column). This effect, which can be clearly seen in Fig. 7(A), provokes a slight drift on the optimal required current for attaining the target RR, which was defined with respect to the initial RR baseline value. However, this RR baseline variability does not affect the control strategy, since the target RR was reached in all cases, and no correlation was found between RR baseline values and the obtained PI performance. Another limitation of this study is related to the model. Although the proposed model is more detailed than those used in previous related studies, it may be improved by a better representation of the electrodenerve interface. Current studies of our team are directed to a better characterization of the autonomic response for different VNS parameters [29].

\section{CONCLUSION}

In this paper, we proposed a model-based framework for the design of control modules for medical devices. This framework allowed us to design PI controllers which work synchronously 
with the heart period, for regulating the heart rate by modifying the stimulation current applied to the VN.

A physiological model representing the CVS of a sheep, including the cardiac response to VNS was used to generate a virtual population. Sensitivity analyses were performed by varying parameters of the control system and the physiological model in order to estimate a domain of interest in the space of the control parameters. The PI controller derived from the proposed MBD approach was experimentally validated on six sheep, by using two different anesthetics. Results clearly show the interest of using a model-based approach in order to determine the parameter values of a PI controller and to optimize the dynamic performance of the closed-loop regulator.

The proposed MBD framework can be generalized to other applications. Our current study is directed to the integration and evaluation of other control methods for the same VNS application (same model of the plant, but different controller), but also to the application of PID-type controllers for the optimization of cardiac resynchronization therapy (same controller, but different plant model). This MBD approach may also help to minimize animal experimentation for the design of closed-loop medical devices.

\section{ACKNOWLEDGMENT}

The authors would like to thank Dr. A. Bel for his constant availability and wise advice, as well as M. Rancic, J. Piquet, and A. Lalot for their contributions on animal experimentation.

\section{REFERENCES}

[1] R. H. Howland, "Vagus nerve stimulation," Curr. Behav. Neurosci. Rep., vol. 1, no. 2, pp. 64-73, 2014.

[2] C. Wu. (2013, Dec.). "Closed-loop stimulation: An investigational treatment for refractory epilepsy," Int. Neuromod. Soc., [Online]. Available: http://www.neuromodulation.com/assets/documents/Fact_Sheets/fact_ sheet_epilepsy_loop.pdf

[3] M. Tosato et al., "Closed-loop control of the heart rate by electrical stimulation of the vagus nerve," Med. Bio. Eng. Comput., vol. 44, no. 3, pp. 161-169, 2006.

[4] H. P. Buschman et al., "Heart rate control via vagus nerve stimulation," Neuromodulation, vol. 9, no. 3, pp. 214-220, 2006.

[5] Y. Zhou et al., "An implanted closed-loop chip system for heart rate control: System design and effects in conscious rats," J. Biomed. Res., vol. 24, no. 2, pp. 107-114, 2010.

[6] H. M. R. Ugalde et al., "On-off closed-loop control of vagus nerve stimulation for the adaptation of heart rate," in Proc. IEEE Eng. Med. Biol. Soc. Int. Conf., Aug. 2014, pp. 6262-6265.

[7] M. S. Waninger et al., "Electrophysiological control of ventricular rate during atrial fibrillation," Pacing Clin. Electro, vol. 23, no. 8, pp. 1239-1244, 2000.

[8] Y. Zhang et al., "Optimal ventricular rate slowing during atrial fibrillation by feedback av nodal-selective vagal stimulation," Amer. J. Physiol. Heart Circ. Physiol., vol. 282, no. 3, pp. H1102-H1110, 2002.

[9] H. Chen and B. Gao, Nonlinear Estimation and Control of Automotive Drivetrains. New York, NY, USA: Springer-Verlag, 2013.

[10] D. Cieslar et al., "Model based approach to closed loop control of 1-D engine simulation models," Control Eng. Pract., vol. 29, pp. 212-224, 2014.
[11] G. D. Kontes et al., "Intelligent BEMS design using detailed thermal simulation models and surrogate-based stochastic optimization," J. Process Control, vol. 24, no. 6, pp. 846-855, 2014.

[12] D. Faille et al., "Control design model for a solar tower plant," Energy Procedia, vol. 49, pp. 2080-2089, 2014.

[13] D. Riu et al., "Control and design of de grids for robust integration of electrical devices. Application to aircraft power systems," Int. J. Electr. Power Amp; Energy Syst., vol. 58, pp. 181-189, 2014.

[14] K. T. V. Koon et al., "Atrioventricular delay optimization in cardiac resynchronization therapy assessed by a computer model," in Proc. IEEE Comput. Cardiol., 2010, pp. 333-336.

[15] A. I. Hernández et al., "Model-based interpretation of cardiac beats by evolutionary algorithms: Signal and model interaction," Artif. Intell. Med., vol. 26, no. 3, pp. 211-235, 2002.

[16] D. Ojeda et al., "Towards an atrio-ventricular delay optimization assessed by a computer model for cardiac resynchronization therapy," presented at the SPIE 8922 IX Int. Seminar Medical Information Processing Analysis, Mexico city, Mexico, 2013.

[17] D. Ojeda et al., "Sensitivity analysis and parameter estimation of a coronary circulation model for triple-vessel disease," IEEE Trans. Biomed. Eng., vol. 61, no. 4, pp. 1208-1219, Apr. 2014.

[18] D. Ojeda et al., "Analysis of a baroreflex model for the study of the chronotropic response to vagal nerve stimulation," in Proc. IEEE/EMBS 7th Int. Neural Eng. Conf., Apr. 2015, pp. 541-544.

[19] B. Smith et al., "Simulation of cardiovascular system diseases by including the autonomic nervous system into a minimal model," Comput. Methods Programs Biomed., vol. 86, no. 2, pp. 153-160, 2007.

[20] V. L. Rolle et al., "Embedding a cardiac pulsatile model into an integrated model of the cardiovascular regulation for heart failure follow-up," IEEE Trans. Biomed. Eng., vol. 58, no. 10, pp. 2982-2986, Oct. 2011.

[21] A. I. Hernández et al., "Integration of detailed modules in a core model of body fluid homeostasis and blood pressure regulation," Progress Biophys. Molecular Biol., vol. 107, no. 1, pp. 169-182, 2011.

[22] M. Ursino and E. Magosso, "Acute cardiovascular response to isocapnic hypoxia. i. a mathematical model," Amer. J. Physiol. Heart Circul. Physiol., vol. 279, no. 1, pp. H149-H165, 2000.

[23] W. Gersch and E. Dong, "A note on Warner's vagus heart rate control model," IEEE Trans. Biomed. Eng., vol. BME-20, no. 2, pp. 145-148, Mar. 1973.

[24] J. C. Helton et al., "A comparison of uncertainty and sensitivity analysis results obtained with random and latin hypercube sampling," Reliab. Eng. Syst. Safety, vol. 89, no. 3, pp. 305-330, 2005.

[25] R. Iman and W. Conover, "Small sample sensitivity analysis techniques for computer models, with an application to risk assessment," Commun. Stat. Theory Meth., vol. A9, no. 17, pp. 1749-1842, 1980.

[26] H. M. R. Ugalde et al., "Model-based design of control modules for neuromodulation devices," in Proc. IEEE/EMBS 7th Int. Neural Eng. Conf., Apr. 2015, pp. 462-465.

[27] D. Andreu et al., "A distributed architecture for activating the peripheral nervous system," J. Neural Eng., vol. 6, no. 2, p. 026001, 2009.

[28] O. Miranda-Domínguez et al., "Firing rate control of a neuron using a linear proportional-integral controller," J. Neural Eng., vol. 7, no. 6, p. 066004, 2010.

[29] L. Rousselet et al., "Influence of vagus nerve stimulation parameters on chronotropism and inotropism in heart failure," in Proc. IEEE Int. 36th Annu. Eng. Med. Biol. Soc., Aug. 2014, pp. 526-529. 